

\title{
Urine Bacteriology in Post-Kidney Transplant Patients with Double-J Stents
}

\section{Abu S1, Igbokwe MC ${ }^{* 1}$, Olatise $\mathrm{OO}^{2}$, Okafor $\mathrm{M}^{1}$, Asaolu SO${ }^{3}$,} Adetunbi AR ${ }^{1}$

\author{
1Urology Division, Zenith Medical and Kidney Centre, Abuja \\ 2Nephrology Unit, Zenith Medical and Kidney Centre, Abuja \\ ${ }^{3}$ Clinical Research Department, Zenith Medical and Kidney Centre, Abuja
}

*Correspondence: Dr MC Igbokwe, Urology Division, Zenith Medical and Kidney Centre, Gudu, Abuja.

E-mail: martin.igbokwe@npmcn.edu.ng; ORCID - https://orcid.org/0000-0002-9155-3262.

\begin{abstract}
Background: Kidney transplantation is the gold standard treatment modality for patients with end-stage renal disease. Ureteric stenting is commonly used during kidney transplantation to reduce the incidence of ureteric complications post-transplantation. The presence of ureteric stents could be complicated by bacterial colonisation and urinary tract infections.

Objective: To identify the urinary flora in patients with double-J stents following kidney transplantation and establish bacteria colonisation and their antimicrobial susceptibility.

Methods: Over one-year, single urine samples of consecutive 100 post-renal transplant patients were subjected to bacteriologic analysis. Early morning midstream urine was obtained into a sterile bottle from all the participants for laboratory analysis.

Results: The mean age of post kidney transplantation patients was $47.6 \pm 12.3$ years. Hypertension and diabetes were the commonest co-morbidities associated with End-Stage-Renal-Disease (ESRD), accounting for 61\% and 28\%, respectively. E. coli was the commonest isolate (70.4\%). Microbiological evidence of Urinary Tract Infection (UTI) revealed by pyuria (pus cells $>4 / \mathrm{HPF}$ ) was found in $40.9 \%$. Tigecycline, nitrofurantoin and tetracycline showed the highest sensitivity pattern in $9 \%, 8 \%$ and $8 \%$, respectively, with significant resistance against cephalosporins and fluoroquinolones.

Conclusion: The fourth week of double-J ureteric stent insertion in kidney transplant recipients showed a high incidence of urinary bacterial colonisation.
\end{abstract}

Keywords: Bacterial colonisation, End-Stage Renal Disease, Double-J, Ureteric stenting, Urinary Tract Infection.

\section{Introduction}

End-Stage Renal Disease (ESRD) affects many people globally, and there is a rise in its prevalence in Nigeria. ${ }^{[1,2]}$ Kidney transplantation remains the gold standard renal replacement therapy for the teeming population of patients with ESRD. [3] It provides the best outcome for these patients as it is cost-effective and increases the quality of life and life expectancy. ${ }^{[3,4]}$ In the developing world, transplant is performed with about $85-100 \%$ of the kidneys from living donors, while in the developed countries, approximately 
$75 \%$ of the transplants performed use kidneys from cadaveric donors. [5] There have been a lot of advances in the use of immunosuppressive treatment with improved graft survival rates. However, infectious complications are yet to improve, with the urinary tract being the most common infection site.

A prevalence of up to $60 \%$ of urinary tract infections among renal transplant patients has been reported in the first year post-transplant. [6, 7] Apart from using immunosuppressive medications, other risk factors include: urological abnormalities, vesicoureteral reflux (VUR), invasive diagnostic and therapeutic procedures involving the urinary tract and the presence of ureteric stents. [7] Urinary tract infections (UTI) can be a source of burden to post-transplant patients as they can negatively affect survival and overall patients' outcome. Ureteric stenting is performed commonly during renal transplantation to reduce the chances of urologic complications like ureteric leak and stenosis. The most frequently used ureteric stent in urological practice globally is the double-J stent. It is a simple, self-retaining polyurethrane material used following a wide range of ureteric and kidney surgeries. However, double-J stents are not without potential complications, including bacterial colonisation and UTI. [8, 9] For this reason, some urologists and kidney transplant surgeons do not advise routine ureteric stents for all patients but rather in selected cases. ${ }^{[8,9]}$ In practice, double-J ureteric stents are used for all kidney transplant recipients and removed four weeks after surgery. This practice aims to reduce the incidence of ureteric stenosis and urine leakage, which occur early following kidney transplantation and not leaving the stents in place for too long to avoid stent-related UTIs or encrustations. There is a lack of data on the urine flora in patients with double-J stents in subSaharan Africa and the incidence of UTIs in post kidney transplant African patients.
This study aims to identify the urinary flora in patients with double-J stents following kidney transplantation and establish bacterial colonisation and their antimicrobial susceptibility.

\section{Methods}

This study is a cross-sectional, descriptive analysis of a series of consecutive post renal transplant patients with indwelling ureteral stents, who came for stents removal at four weeks post-transplantation at the Zenith Medical and Kidney Centre, Abuja, North-central Nigeria. The hospital is a 100-bed super-specialised kidney centre that receives patients from all parts of Nigeria. There are two kidney surgeons resident in the facility. An average of 12 renal transplants are performed in the centre in a month.

The patient is placed in the supine position for kidney transplantation surgery, and general or regional anaesthesia is administered. A Gibson's incision is made and deepened in a complete extra-peritoneal fashion to access the iliac group of vessels and the ipsilateral ureter. The external iliac artery and vein are dissected and mobilised to allow easy, tension-free anastomosis. A bookwater self-retaining retractor enables excellent exposure during anastomosis of the renal allograft artery and vein to the iliac vessels as well as the ureteroneocystostomy/ureteroureterostomy. Vascular anastomosis is carried out using prolene $5 / 0$ or $6 / 0$ sutures using the external or internal iliac artery or vein. Ureteroneocystostomy is done using PDS 4/0 sutures with the modified Lich Gregoir technique or end-to-side uretero-ureterostomy over a double-J stent and wound drains inserted. The urine culture of all patients for kidney transplantation is sterile before transplantation.

The hospital's Health Research Ethics Committee approved the study. This study of 100 patients 
from our institution was conducted between January and December 2020. All the patients gave informed consent before the collection of urine specimens. Patients with urine leaks, allograft dysfunction, or vascular complications and those with bladder outlet obstruction were excluded from this study.

Data (such as age and medical history) of kidney transplant recipients were obtained from the electronic medical records. Early morning midstream urine samples were obtained into sterile bottles from all the participants within the hospital laboratory premises following adequate counselling on the collection technique. Only one urine sample was collected from each patient. The time frame between the collection and centrifugation of the urine sample was four hours. The urine sample was centrifuged and prepared on a slide for microscopy using a simple light microscope (OPTIKA B-380 series, Optika Microscopes, Italy.). The urine sample was also inoculated onto blood agar and eosin methylene blue agar. Plates were incubated for 48 hours at $37^{\circ} \mathrm{C}$. The microorganisms that grew on the agar were evaluated quantitatively. Bacteria were identified by conventional methods. All microbial isolates were tested for their susceptibility to a panel of 19 antibiotics. The efficacy of the antibiotics was determined by the measurement of their zones of inhibition. All the specimens were handled according to the Clinical Microbiology Laboratory Standard Operating Procedures.

Data analysis was performed using SPSS version 17 for Windows (SPSS Inc., Chicago, IL, USA). Categorical variables are expressed as frequency and percentages, while the Chi-Square test was used to test for association between categorical variables. Associations were considered statistically significant when $\mathrm{p}<0.05$.

\section{Results}

A total of 100 hundred post-transplant patients (comprising $69(69.0 \%)$ males and 31 (31.0\%) females) were studied. The mean age of the patients was $47.6 \pm 12.3$ years. The mean interval between transplant and recruitment into the study was $4.2 \pm 1.2$ weeks.

The co-morbidities found among the post-kidney transplant patients are shown in Figure 1. In all, fifty-four $(54 \%)$ of the culture grew isolates. The bacterial isolates in the urine samples included: Escherichia coli, Pseudomonas aeruginosa., Staphylococcus aureus, Klebsiella and Streptococci, as shown in Table I.

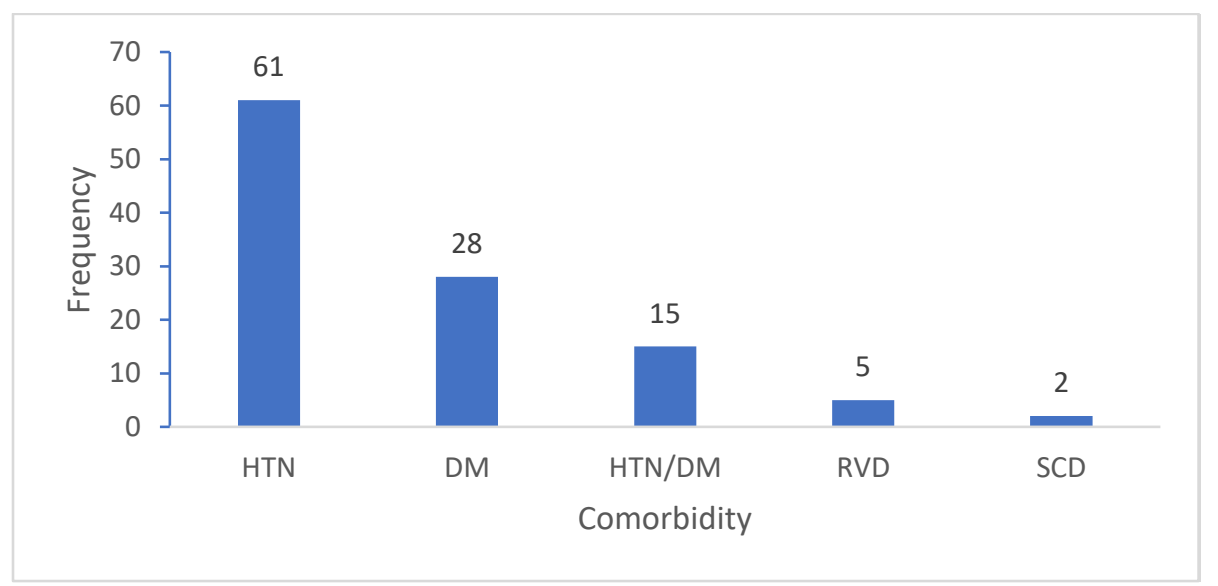

Figure 1: Frequency of co-morbidities among post-kidney transplant patients

HTN - Hypertension; DM - Diabetes mellitus; SCD - Sickle Cell Disease; RVD - Retroviral Disease 
Table I: Organisms isolated in the urine samples of post-kidney transplant patients

\begin{tabular}{lll}
\hline Bacteria & Frequency & Percentage \\
\hline Escherichia coli & 38 & 70.4 \\
Pseudomonas spp. & 8 & 14.8 \\
Staphylococcus aureus & 4 & 7.4 \\
Klebsiella spp. & 2 & 3.7 \\
Streptococcus & 2 & 3.7 \\
\hline
\end{tabular}

There was no relationship between urine colonisation and age or gender $(p=0.204)$. Those with less than four pus cells / HPF accounted for $59.1 \%$, while $40.9 \%$ had pus cells greater than four /HPF. Out of the 100 urine samples, 46 $(46.0 \%)$ showed no bacterial growth. Out of the 28 patients with diabetes mellitus, $13(46.4 \%)$ had no bacterial growth. Also, out of the 72 patients without diabetes mellitus, 33 (45.8\%) had no bacterial growth. However, there was no statistical significance between diabetes and bacteria culture $(\mathrm{p}=0.709)$.

Regarding the bacteria sensitivity pattern, tetracycline showed the highest sensitivity (7\%) to $E$. coli. This was closely followed by tigecycline $(6 \%)$, while nitrofurantoin $(4 \%)$, levofloxacin $(4 \%)$ and cephalexin $(4 \%)$ are on the same level as shown in Table II. Interestingly, Table III shows that cefotaxime had the highest resistance $(21.0 \%)$ to the isolates compared to other tested antibiotics.

\section{Discussion}

Double-J stents in kidney transplant recipients significantly reduce urologic complications of transplant surgery such as urine leaks and ureteric stenosis. [10, 11] However, it may be associated with a few complications such as suprapubic pain, vesicoureteric reflux, stent migration, encrustation, stent fracture, and urinary infections hence the rationale for using the procedure in selected patients. $[10,11]$

Table II: Pattern of sensitivity of the bacterial isolates

\begin{tabular}{llllll}
\hline Drugs & \multicolumn{5}{c}{ Bacteria } \\
\hline Tigecycline & E. coli & S. aureus & Klebsiella & Pseudomonas & Streptococcus \\
Nitrofurantoin & 4 & 2 & 1 & 0 & 0 \\
Tetracycline & 7 & 2 & 0 & 1 & 1 \\
Ciprofloxacin & 3 & 0 & 0 & 0 & 0 \\
Levofloxacin & 4 & 2 & 0 & 1 & 0 \\
Cephalexin & 4 & 0 & 0 & 0 & 0 \\
Gentamycin & 3 & 0 & 0 & 0 & 0 \\
Erythromycin & 0 & 1 & 0 & 1 & 0 \\
Amoxylclauf & 0 & 2 & 0 & 0 & 0 \\
Nalidixic acid & 1 & 1 & 0 & 0 & 0 \\
Ceftriaxone & 0 & 2 & 0 & 0 & 0 \\
Amoxil & 0 & 1 & 0 & 0 & 0 \\
Ofloxacin & 0 & 1 & 0 & 0 & 0 \\
Cefuroxime & 1 & 0 & 0 & 0 & 0 \\
Cefotaxime & 0 & 1 & 0 & 0 & 0 \\
\hline \multicolumn{1}{l}{ Figures represent }
\end{tabular}

Figures represent the percentage susceptibilities of the isolates to the various antibiotics. 
Table III: Pattern of resistance of the bacterial isolates

\begin{tabular}{llllll}
\hline Drugs & & \multicolumn{4}{c}{ Bacteria } \\
\hline Ceftriaxone & E. coli & S. aureus & Klebsiella & Pseudomonas & Streptococcus \\
Clindamycin & 20 & 1 & 2 & 8 & 1 \\
Erythromycin & 17 & 2 & 2 & 7 & 0 \\
Cefotaxime & 21 & 1 & 1 & 6 & 1 \\
Nalidixic acid & 18 & 2 & 1 & 3 & 0 \\
Levofloxacin & 19 & 0 & 2 & 5 & 0 \\
Tetracycline & 17 & 2 & 1 & 4 & 1 \\
Ciprofloxacin & 19 & 1 & 0 & 4 & 1 \\
Cefuroxime & 13 & 1 & 0 & 5 & 0 \\
Gentamycin & 10 & 0 & 2 & 5 & 1 \\
Amoxil & 12 & 0 & 1 & 2 & 1 \\
Trimethoprim & 11 & 1 & 0 & 2 & 1 \\
Ofloxacin & 13 & 0 & 0 & 0 & 0 \\
Nitrofurantoin & 10 & 0 & 0 & 3 & 0 \\
Amoxylclauf & 8 & 0 & 0 & 1 & 0 \\
Cephalexin & 6 & 1 & 0 & 0 & 0 \\
Cefixime & 1 & 0 & 0 & 0 & 0 \\
Tigecycline & 0 & 0 & 0 & 1 & 0 \\
Co- & 0 & 0 & 0 & 1 & 0 \\
trimoxazole & & & & & 0 \\
\hline \multicolumn{1}{l}{ Figurs }
\end{tabular}

Figures represent the percentage resistance of the isolates to the various antibiotics.

This study showed a high prevalence of urine colonisation in post-kidney transplant patients with double-J stents in our practice. This could be attributable to several factors, including the foreign body effect of the double-J stent, the relatively immunosuppressive state of chronic kidney disease and the use of anti-rejection drugs like steroids to reduce the recipient's immunity. Despite the higher rate of bacterial colonisation in this cohort of patients, the incidence of urinary tract infection as evidenced by white blood cells/pus cells in urine was not very high_at the time of sampling and stent removal.

There was a higher population of male participants in this study, which may reflect the higher male population among kidney transplant recipients in Nigeria. [12, 13] The mean age of patients in this study was higher than in the study by Shohab et al. in Pakistan, where the mean age of participants was $34.01 \pm 14.63$ years. [8] The decision to remove the double J stents at four weeks post-kidney transplant is a unit protocol and was similarly recommended by Tavakoli et al., who found a higher incidence of urinary tract infections (UTI) when double-J stents stayed beyond four weeks in these group of patients. [14]

As reported widely in literature, hypertension and diabetes mellitus were the commonest comorbidities among renal transplant patients, being the main risk factors for ESRD. [2,15,16] E. coli was the most frequently isolated microbe in the urine of these patients accounting for 7 in 10 positive cultures. This was comparable to the findings in some other studies. $[17,18]$ On the other hand, Kozyrakis et al. found Staphylococcus aureus as the commonest isolate in their research. ${ }^{[19]}$ It was also remarkable that $46 \%$ of the patients had no bacterial isolate in their urine samples. However, the general resistance of the bacterial microbes to antibiotics on the tray and a poor sensitivity pattern, especially to fluoroquinolones 
and cephalosporins, which are the most commonly used antibiotics for UTIs, is worrisome. This observation aligns with the global trend of multi-drug resistance among pathogens of UTIs. [20, 21]

Despite the relatively high colonisation rate of the urine in patients with double-J stents in this study, the rate of UTIs based on the WHO definition of UTIs was significant. This suggests that the body's immunity can still curtail bacterial colonisation at this stage or that removing stents within four weeks is ideal, at which time stentrelated UTIs are not likely to be established. However, the inability to culture anaerobes and fungi significantly limits this study.

\section{Conclusion}

Double-J stents in kidney transplant patients can be associated with urine bacterial colonisation in a significant population of patients. E. coli is the commonest organism found in the urine of this group of patients, and the commonest antibiotics to which the isolates were sensitive were tigecycline, nitrofurantoin and tetracycline. There is a need to assay the microbiological flora on the double-J stents for a more comparative study.

Acknowledgement: The authors appreciate the entire theatre staff and laboratory staff of the Zenith Medical and Kidney Centre, Abuja, who contributed to the success of this study.

Authors' Contributions: AS and IMC conceived the study. AS, IMC and AAR designed the study. All the authors did literature review. AS, OM, ASO and AAR analysed the data while IMC, AS, OM, ASO, and AAR interpreted the data. $\mathrm{OOO}$ and ASO drafted the manuscript, IMC and OOO revised the draft for sound intellectual contents. All the authors approved the final draft of the manuscript.

Conflicts of Interest: None.

Funding: Self-funded.
Publication History: Submitted 22 July 2021; Accepted 12 November 2021.

\section{References}

1. Garcia GG, harden P, Chapman J. the global role of kidney transplantation. Am J Nephrol 2012; 35: 259-264.

https://doi.org/10.5812/nephropathol.7448

2. Salako AA, Badmus TA, Igbokwe MC, David RA, Laoye A, Akinbola IA, et al. Experience with arteriovenous fistula creation for maintenance hemodialysis in a tertiary hospital in South-western Nigeria. Saudi J Kidney Dis Transpl 2018; 29: 924-929. https://doi.org/10.4103/1319-2442.239628

3. Jha V. Current status of end-stage renal disease care in South Asia. Ethn Dis 2009; 19; 27-32.

4. Dulal RK, Karki S. Nepalese kidney transplant recipient in a follow-up clinic: related and unrelated living donor. J Nepal Med Assoc 2008; 47: 98-103.

5. Enns J, Aryal G. A path to renal transplantation in Nepal. J Pathol Nepal 2011; 1: 52-55.

6. Veroux M, Giuffrida G, Corona D, Gagliano M, Scriffignano V, Vizcarra D. Infective complications in renal allograft recipients: Epidemiology and outcome. Transplantation Proc 2008; 40: 1873-1876. https://doi.org/10.1016/j.transproceed.2008.05 $\underline{.065}$

7. Golębiewska J, Dębska-Ślizień A, Komarnicka J, Samet A, Rutkowski B. urinary tract infections in renal transplant recipients. Transplantation Proc 2011; 43: 2985-2990. https:// doi.org/10.1016/j.transproceed.2022.07 $\underline{.010}$

8. Shohab D, Khawaja A, Atif E, Jamil I, Ali I, Akhter S. Frequency of occurrence of urinary tract infection in double J stented versus nonstented renal transplant recipients. Saudi J Kidney Dis Transpl 2015; 26: 443-446. https:// doi.org/10.4103/1319-2442.157298 
9. Kirnap M, Boyvat F, Torgay A, Moray G, Yildirim S, Haberal M. Incidence of urinary complications with Double J Stents in Kidney Transplantation. Exp Clin Transplant 2019; 1: 148-152. https://doi.org/10.6002/ect.MESOT2018.P14

10. Indu KN, Lakshminarayana G, Anil M. Is early removal of prophylactic ureteric stents beneficial in live donor renal transplantation? Indian J Nephrol 2012; 22: 275-279. https://doi.org/10.4103/0971-4065.101247

11. Shabeena KS, Bhargava R, Manzoor MAP, Mujeeburahiman M. Characteristics of bacterial colonisation after indwelling double-J ureteral stents for different time duration. Urol Ann 2018; 10: 71-75. https://doi.org/10.4103/UA.UA_158_17

12. Igbokwe MC, Abu S, Aremu A, Olatise O, Okafor M. Spectrum of Endo-urological procedures performed at a Nigerian Kidney Transplant Centre. Yenagoa Med J 2020; 2: 1-6.

13. Igbokwe M. Experience and early outcomes of Kidney Transplantation in a Nigerian Transplant in a Nigerian Transplant Institution: A 1-Year Review. 40 $0^{\text {th }}$ Congress of the Societe Internationale D'Urologie; montreal, Canada: SIU Academy; 2020. pSP-18.02. https://doi.org/10.13140/RG.2.2.30933.14562

14. Tavakoli A, Surange RS, Pearson RC, Parrott NR, Augustine T, Riad HN. Impact of stents on urological complications and health care expenditure in renal transplant recipients: Results of a prospective, randomised clinical trial. J Urol 2007; 177: 2260-2264. https://doi.org/10.1016/j.juro.2007.01.152
15. Arogundade FA. Kidney transplantation in a low-resource setting: Nigeria Experience. Kidney Int Suppl 2013; 3: 241-245. https://doi.org/10.1038/kisup.2013.23

16. Ulasi II, Ijoma CK. The enormity of chronic kidney disease in Nigeria: the situation in a teaching hospital in southeast Nigeria. J Trop Med 2010; 2010.501957.

https://doi.org/10.1155/2010/501957

17. Joshi R, Singh DR, Sharma S. Lower urinary tract infection and bacterial colonisation in patients with Double-J ureteral stent. J Nepal Health Res Counc 2011; 9: 165-168.

18. Kehinde EO, Rotimi VO, Al-Hunayan A. Bacteriology of urinary tract infection associated with indwelling J ureteral stents. J Endourol 2004; 18: 891-896. https://doi.org/10.1089/end.2004.18.891

19. Kozyrakis D, Perikleous $S$, Chatzistamou Z-E, Kateris D, Soukias G, Karatzas A, et al. Is there a role for Double J stent culture in contemporary urology? Urol Int 2018; 100: 203208. https://doi.org/10.1159/000486798

20. Nzalie RN-t, Gonsu HK, Koulla-Shiro S. Bacterial etiology and antibiotic resistance profile of community-acquired urinary tract infections in a Cameroonian city. Int J Microbiol 2016; 2016: 3240268. https://doi.org/10.1155/2016/3240268

21. Ahmed SS, Shariq A, Alsalloom AA, Babikir $\mathrm{IH}$, Alhoumoud BN. Uropathogens and their antimicrobial resistance patterns: Relationship with urinary tract infections. Int J Health Sci 2019; 13: 48-55. unrestricted, non-commercial use, reproduction and distribution in any medium provided the original source is adequately cited and credited. 